Revue des patrimoines

19 | 2012

Patrimoines et conservation préventive. Pratiques comparées et nouveaux enjeux

\title{
Conservation préventive au Musée Crozatier : vers une renaissance assurée
}

Isabelle Boiché et Emmanuel Magne

\section{(2) OpenEdition}

Journals

Édition électronique

URL : http://journals.openedition.org/insitu/9823

DOI : $10.4000 /$ insitu.9823

ISSN : 1630-7305

Éditeur

Ministère de la culture

Référence électronique

Isabelle Boiché et Emmanuel Magne, « Conservation préventive au Musée Crozatier : vers une renaissance assurée », In Situ [En ligne], 19 | 2012, mis en ligne le 21 septembre 2012, consulté le 03 mai 2019. URL : http://journals.openedition.org/insitu/9823 ; DOI : 10.4000/insitu.9823

Ce document a été généré automatiquement le 3 mai 2019.

\section{cc)}

In Situ Revues des patrimoines est mis à disposition selon les termes de la licence Creative Commons Attribution - Pas d'Utilisation Commerciale - Pas de Modification 4.0 International. 


\section{Conservation préventive au Musée Crozatier : vers une renaissance assurée}

Isabelle Boiché et Emmanuel Magne

\section{Le musée Crozatier en 2002 : état des lieux des collections et des coulisses}

Figure 1

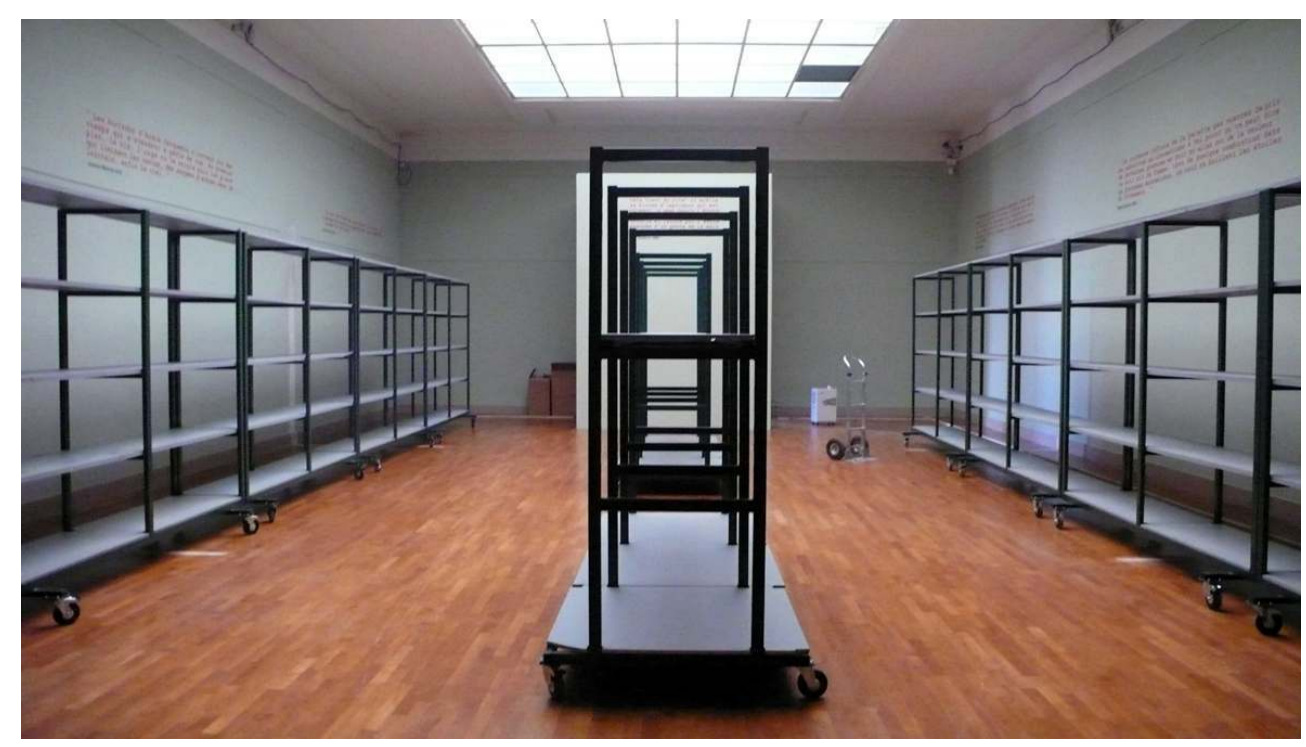

En prévision du chantier des collections : des espaces et mobiliers en préparation.

(c) Boiché, Isabelle, IBM Conservation, 2009.

1 Le musée Crozatier est riche de mille collections qui en font un des rares exemples de musée encyclopédique encore conservé en France (fig. $\mathbf{n}^{\circ} \mathbf{1}$ ). Fondé en 1820 et 
constamment enrichi de dons, d'achats et de dépôts de l'État, il conserve plus de 70000 objets de la statue en marbre jusqu'au mortier en bronze en passant par le lapidaire, les artisanats locaux, l'archéologie, les beaux-arts, les sciences et techniques, les sciences naturelles sans oublier les 100000 items liés à la dentelle. Ce n'est pas un musée mais plusieurs musées que contient le bâtiment érigé dans le jardin public grâce au legs du fondeur d'art Charles Crozatier en $1868^{1}$ (fig. $\mathbf{n}^{\circ} \mathbf{2}$ ).

Figure 2

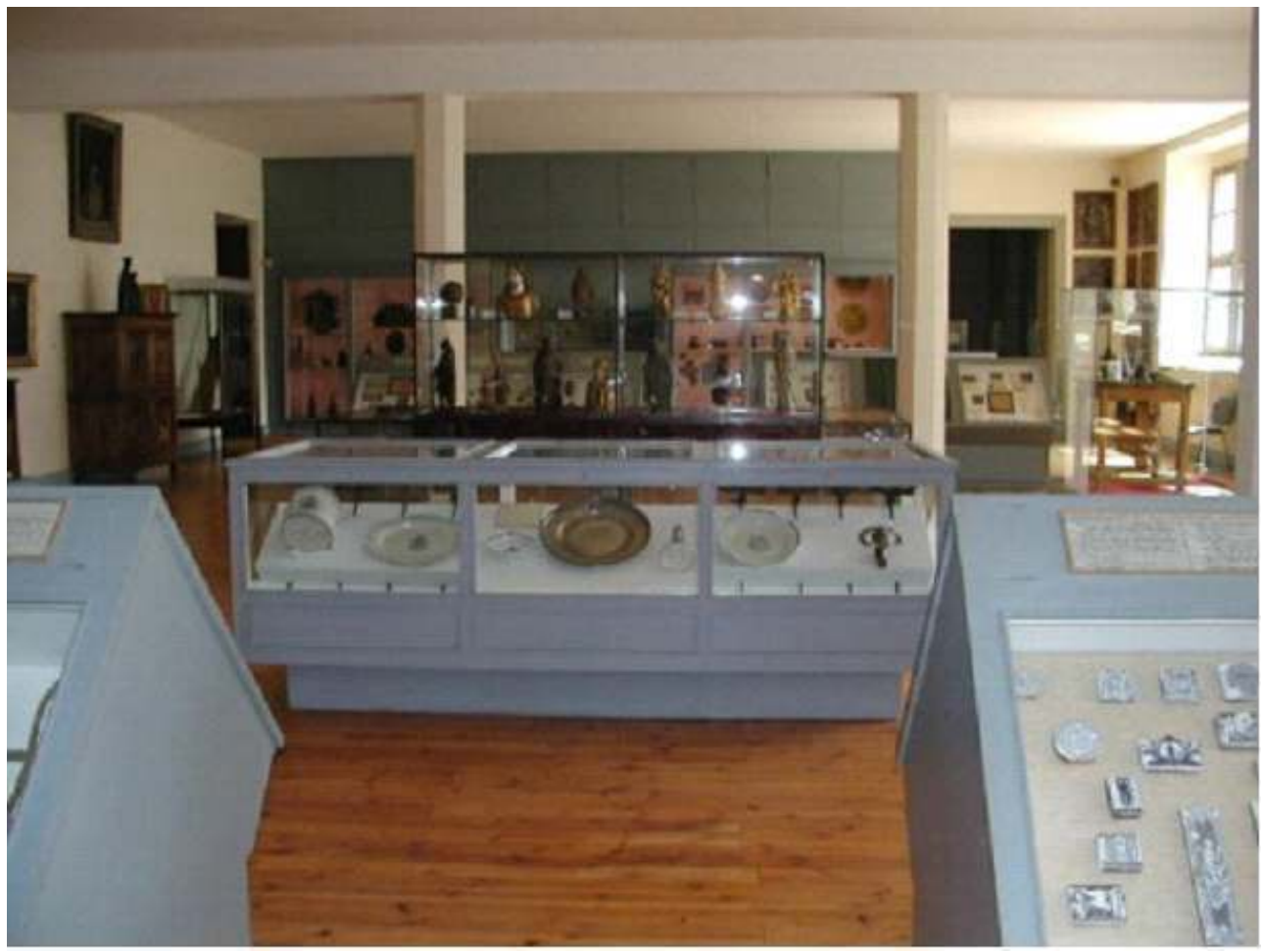

Salle d'exposition.

(c) Boiché, Isabelle, IBM Conservation, 2008.

2 Réaménagé dans les années 1950, le musée n'avait alors pas fait l'objet d'une remise à niveau de son infrastructure. En 2002, le musée s'organisait donc sur cinq niveaux (soit $4588 \mathrm{~m}^{2}$ ), les combles et le niveau inférieur étant principalement affectés aux réserves ( $930 \mathrm{~m}^{2}$ soit moins de $20 \%$ du musée). Les salles d'exposition représentaient une surface de $2400 \mathrm{~m}^{2}$ (hauteur sous plafond: de 3,60 à $11 \mathrm{~m}$ selon les salles) soit plus de $50 \% \mathrm{du}$ musée.

3 Si des expositions temporaires étaient organisées régulièrement au rez-de-chaussée et bénéficiaient d'aménagements contemporains, les autres salles connaissaient une muséographie vieillissante.

4 Le visiteur explorait donc un parcours muséographique très contraignant, au sein d'espaces non climatisés, non chauffés, sans ascenseur, dépourvus d'équipements de détection incendie, bref dans des conditions ne répondant plus aux normes contemporaines...

5 Consciente de ce lourd passif, la municipalité du Puy-en-Velay a entrepris un important chantier de rénovation prévoyant la reconstruction du bâtiment arrière du musée, ce qui permettra de le doter de tous les équipements nécessaires au fonctionnement normal 
d'un établissement accueillant du public. Parallèlement toute la muséographie va être repensée, même si certaines galeries conserveront un esprit ancien, témoin de l'histoire du musée.

\section{2 : l'élan de la conservation préventive}

6 Confrontée à ces conditions patrimoniales peu performantes, l'équipe du musée Crozatier a entamé, à partir de 2002 et jusqu'en 2011, différentes missions en conservation préventive pour construire un nouvel outil de valorisation d'un patrimoine local de référence.

Dans ce cadre, différents temps forts ont été impulsés, depuis le bilan sanitaire global des collections jusqu'aux dernières opérations de récolement, prises de vue et conditionnement, en passant par des campagnes de formation de personnel, réalisation de campagne de dépoussiérage, etc.

Toute une chaîne opératoire s'est donc progressivement mise en place pour améliorer durablement la conservation et la connaissance des collections du musée, transformant peu à peu le musée en un site de référence dans le monde des musées.

9 Nous insisterons dans le cadre de cet article sur la dynamique du chantier des collections ayant mobilisé une grande partie de l'équipe du musée entre 2007 et 2010, une année de fermeture au public du musée...

\section{Chantier des collections : redécouverte des collections, mise en place de nouvelles procédures de travail}

10 Dans une logique de préparation aux travaux de rénovation, le chantier des collections devait progressivement se mettre en place pour permettre une mise en conservation sécurisée des collections avant le lancement des travaux. Précisons que le projet initial prévoyait la rénovation du musée en dehors de la présence des collections : ce contexte n'a finalement pas été retenu, les collections devant rester in situ et déménager d'une salle à l'autre en fonction du phasage des travaux...

11 La volonté affirmée a été de confier le lancement et la supervision du chantier, conduit exclusivement en interne, à un mandataire spécialisé en conservation préventive expérimenté. En effet, il avait été décidé qu'une grande partie de l'équipe du musée (environ une dizaine de personnes en fonction des périodes) devait se consacrer aux différentes opérations propres à un chantier de collections : dépoussiérage, récolement et informatisation, prise de vue, marquage, conditionnement. Dès lors, en 2007 s'est amorcée la mise en place d'une chaîne opératoire complexe perturbant les habitudes de travail. Jusqu'à présent, les agents avaient pour habitude de travailler à des postes bien précis : agents d'accueil et de surveillance et personnel technique avaient peu l'habitude de travailler ensemble. Or la mise en place du chantier les amena à partager espaces de travail et missions de mise en conservation. 


\section{La connaissance des inventaires et des sources d'archives}

12 La chaîne opératoire mise en œuvre comprenait l'étape du récolement qui au musée n'avait jamais été fait. Or entreprendre le récolement d'une collection aussi étendue et diversifiée est une mission dont on ne mesure la complexité qu'une fois plongé au cœur des inventaires de l'institution. Il est alors nécessaire de comprendre l'histoire du musée et d'appréhender le mode de travail des différents conservateurs qui ont assuré la gestion des collections depuis sa fondation.

13 Jusqu'en 1878, pas ou peu de listes. Les plus complètes concernent les beaux-arts pour lesquels on dispose d'abord de notices éditées en plusieurs fois dans les Annales de la Société Académique du Puy. Un catalogue de cette collection est publié en 1872 grâce au travail de Jean-Baptiste-Pierre Vibert (1800-1871). Cet ouvrage sera repris et complété en 1904 de quelques autres collections par Édouard Terrasse (1856-1924). Ce n'est qu'après 1878 suite à la gestion directe du musée par la municipalité que les premières listes ont pu être dressées par Hippolyte Malègue, conservateur au musée. Celui-ci a consigné, sur deux registres, les objets par types de collections.

Le registre A regroupe : les antiquités lapidaires romanes, gothiques puis gallo-romaines, les sculptures en plâtre, la flore, la collection des gravures, dessins, lithographies, aquarelles et photographies, l'ethnographie, la collection des tableaux de peintures, la collection de serpents et les médailles obtenues dans différents concours par les sociétés musicales.

15 Le registre B liste successivement la collection dite des machines d'Alexandre Clair, l'inventaire de la bibliothèque donnée au musée par Alexandre Clair, la collection préhistorique, l'inventaire du mobilier archéologique (fonderie, faïences et porcelaines, franc-maçonnerie, les sceaux, et objets divers), la collection anatomique et la bibliothèque du musée.

Pour chaque collection, la numérotation repart à zéro, cependant aucun objet n'est marqué. Les désignations sont parfois approximatives voire peu significatives.

17 Des registres sont consacrés aux monnaies et médailles. Ils ont été rédigés de manière très détaillée par Achille Monthélhet, numismate local, qui après avoir donné sa collection de près de 10000 pièces, a rédigé les inventaires de la totalité de la collection.

Deux autres carnets reprennent en détail la collection de préhistoire et nombre d'objets sont cités à la fois dans ces carnets et dans le registre A à la section ethnographie.

L'autre source importante pour connaître les dates et moyens d'entrée des œuvres dans les collections du musée sont les Annales de la société académique qui jusqu'en $1878 \mathrm{a} \mathrm{eu}$ la charge de la gestion des collections. Les comptes-rendus mensuels rapportent les dons faits au musée. Mais là aussi les dénominations sont peu détaillées et les donateurs pas toujours nommés.

\section{L'inventaire normalisé}

20 À partir de 1950, Roger Gounot (1909-1979), conservateur du musée, a, selon les normes édictées alors par la Direction des Musées de France, commencé la rédaction des 
inventaires en marquant les objets et en complétant les 18 colonnes de l'inventaire réglementaire. C'est lui le premier qui a tenté un récolement, mais il a procédé d'une manière inverse à celle que nous pratiquons aujourd'hui : il est parti des sources et non des objets. Ce travail de bénédictin, qu'il a conduit seul, a duré plus de trente ans. Très rigoureux dans sa démarche, Roger Gounot a inscrit dans l'inventaire tous les objets dont les sources mentionnaient l'entrée mais sans parfois pouvoir repérer physiquement l'œuvre. L'intérêt de la démarche est que cet inventaire normalisé constitue un document exhaustif des œuvres et objets qui sont entrés au musée. Le revers est qu'il est difficile de s'en servir de base pour aboutir à un récolement de l'ensemble des collections parce qu'il est aujourd'hui nécessaire de passer chaque objet en revue, pour vérifier sa présence réelle et pour éliminer les doublons.

Ce travail s'est doublé de la constitution d'un fichier matière sur fiches cartonnées qui renvoient au numéro d'inventaire. Ces fiches, dont les entrées mentionnent aussi bien la désignation de l'objet que son donateur ou son lieu de découverte, permettent de croiser les informations pour tenter de trouver un numéro d'inventaire à un objet isolé. Cette documentation a été un de nos outils principaux dans notre travail de récolement et d'inventaire.

\section{Informatisation et récolement}

22 À partir de septembre 1985 et jusqu'en 1987, les collections de peintures, de sculptures et de dessins ont fait l'objet d'une saisie informatique par système de bordereaux sur la base Joconde mise en place par le ministère de la Culture.

En 1998, le musée a acquis un logiciel de gestion des collections de musée qui a permis de poursuivre, en interne, la saisie des données. L'ensemble des bases de données contient plus de 60000 fiches, dont les $2 / 3$ sont dotées d'images numériques.

Nous avons établi une procédure d'intervention pour chaque objet quelle que soit sa valeur historique, scientifique ou esthétique: vérification du numéro d'inventaire, récolement, dépoussiérage, photographie et opérations de conditionnement. À chaque objet nous avons associé un code-barre créé en interne. Celui-ci est imprimé sur une étiquette apposée ou accrochée à l'œuvre de manière a être visible sans déplacer l'objet. Ce code-barre autorise une gestion facilitée du mouvement des œuvres à l'intérieur du musée. En effet, le chantier de rénovation se déroulera en plusieurs phases successives avec déplacement des collections en fonction de l'avancement des travaux. Le principal déménagement est prévu après la livraison du bâtiment arrière et avant le début des travaux sur le bâtiment sur jardin. Cela implique une bascule complète des collections qui sont à ce jour entièrement stockées dans le bâtiment donnant sur le jardin. Chaque objet est localisé dans une caisse, un tiroir, une étagère ou une cimaise qui eux-mêmes sont dotés de code-barres. Quelque soit le niveau de la hiérarchie de localisation, nous pouvons par simple lecture du code-barre faire afficher les caisses et objets qui y sont localisés et leur affecter une nouvelle localisation.

Le travail a commencé par les collections de sciences naturelles. La géologie et les herbiers avaient été inventoriés en 1998 et 1999 par des personnes recrutées pour l'occasion sous la direction d'universitaires reconnus dans leurs domaines. La paléontologie a été inventoriée en 2002 suivant le même principe. Restaient à inventorier les collections de zoologie pour lesquelles nous avions des listes mises à jour grâce à la 
collaboration du musée Lecoq de Clermont-Ferrand. Pour ces collections, le récolement s'est confondu avec l'établissement d'un inventaire normalisé rétrospectif.

Le récolement s'est poursuivi avec les collections de beaux-arts. Suite à des désordres dans les réserves, une partie de la collection avait déjà été vue en 1998. Cela a été l'occasion de faire venir des spécialistes pour confirmer ou proposer des attributions. Ce travail débouchera cette année sur une mise à jour du versement à la base Joconde.

De 2008 à 2010, les opérations de récolement et de conditionnement se déroulaient essentiellement durant les mois d'hiver, d'avril à octobre une grande partie du personnel étant occupée par les missions d'accueil et de surveillance. Pendant les périodes estivales, le personnel des bureaux attaché aux missions de récolement s'occupait des collections d'art graphiques et de photographies.

À partir de 2009, nous avons entamé l'étude des collections de textiles, des objets d'art, d'ethnographie et d'art extra-européen. Pour ces collections, le récolement a été particulièrement difficile car pour nombre d'objets non marqués, il a été difficile de savoir s'ils avaient perdu leur numéro, s'ils n'avaient jamais été inventoriés ou s'ils faisaient partie des objets inscrits par Roger Gounot, mais qu'il n'avait pas vus. Pour les objets pour lesquels aucun rapprochement avec un numéro existant n'était possible, il a été créé un numéro de récolement.

En 2012, nous avons entamé les collections archéologiques que Roger Gounot avait entièrement inventoriées et marquées, même le plus infime tesson de céramique. Parallèlement et depuis 2008, un agent s'est chargé de la collection de numismatique (10 000 numéros environ) pour laquelle nous disposions des catalogues très précis d'Achille Monthelet mais avec un certain désordre dans le rangement des boîtes contenant les monnaies et médailles. Ce travail a été compliqué par le fait qu'un grand nombre de monnaies avait été posé sur un support en bois peint. Avec le temps, la monnaie s'était collée sur la peinture.

\section{Formation du personnel}

Une des bases de réussite d'un chantier des collections est la mise en place d'un cycle de formation par des formateurs spécialisés, associée à un suivi rigoureux et régulier de la part de ces formateurs. Ce pré-requis avait parfaitement été intégré dans les besoins exprimés par la direction du musée. L'équipe du musée dédiée au chantier a bénéficié, en phase de lancement puis régulièrement, de journées de formations pour assimiler de nouvelles méthodes de travail. Ces formations se sont déclinées diversement en tenant compte des spécificités des collections: tableaux, textiles, documents graphiques, Naturalia, fluides en bocaux, etc.

Dans ce cadre, différents restaurateurs sont intervenus ; Catherine Lebret ${ }^{2}$ ayant assuré le suivi sur les tableaux et Isabelle Boiché ${ }^{3}$ sur le reste de la collection, chacune venant au musée suivant les nécessités du chantier.

Ces formations ont donné lieu à la rédaction de fiches de procédures de traitements adaptés aux modes de travail validés sur site de façon à constituer une ressource documentaire spécifique propre au musée. 


\section{Dépoussiérage des collections}

Le musée, sur les conseils des prestataires, a investi dans différents équipements techniques pour conduire les opérations d'assainissement et de conservation des collections.

4 Le dépoussiérage a été réalisé systématiquement sur les collections, en fonction de procédures envisagées lors des formations. Peu à peu, l'équipe s'est rendue autonome et a appris à distinguer les œuvres manipulables et gérables en interne de celles à confier à l'observation d'un restaurateur.

Dans le cadre du dépoussiérage, un constat sommaire était réalisé pour permettre d'enregistrer sur le logiciel d'inventaire les éléments fondamentaux.

En cas de doute, ces objets étaient mis de côté en prévision d'un examen ou d'une validation par Isabelle Boiché.

\section{Conditionnement des collections}

Une fois dépoussiéré, chaque objet était conditionné selon des procédures évoquées en formation. Dans la mesure du possible, l'équipe a réalisé des conditionnements pérennes au sein de tiroirs ou de bacs gerbables (fig. $\mathbf{n}^{\circ} \mathbf{3}$ ) sans perdre de vue la contrainte de mobilité des contenants au sein d'un musée devant vivre différentes phases de travaux. Les solutions adoptées devaient donc permettre, outre un stockage définitif, de répondre à la nécessité de déplacer les ensembles au fur et à mesure de l'avancée des travaux !

Figure 3

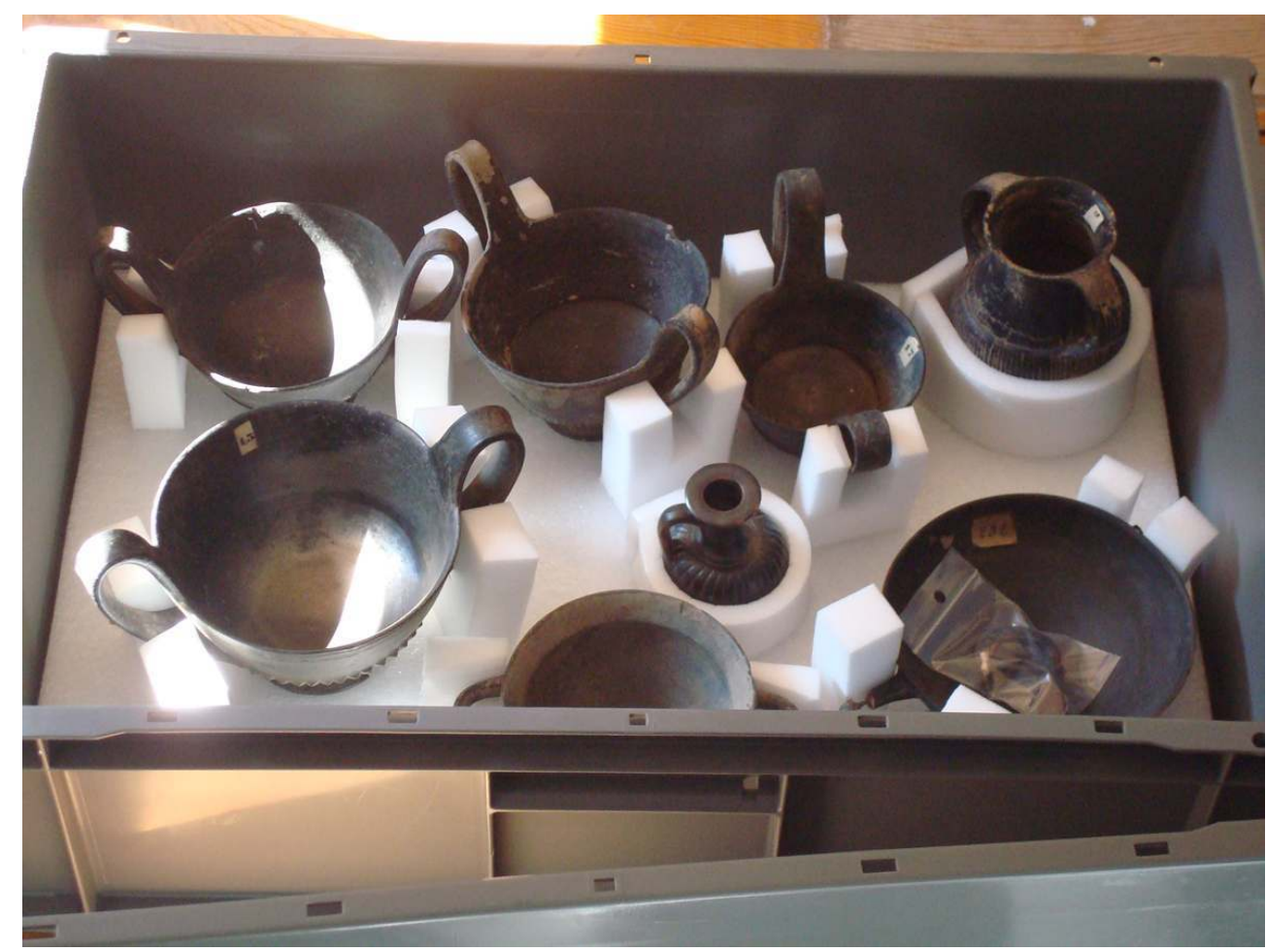

Calage sur mesure pérenne avant mise en bacs.

(c) Boiché, Isabelle, IBM Conservation, 2009. 
reflexion sur le choix des modes de conditionnement a largement été guidée par l'expérience d'Isabelle Boiché sur les collections d'un grand musée parisien où, durant deux ans, sa propre équipe a réalisé des conditionnements ${ }^{4}$ sur mesure répondant à des contraintes fortes : calage pérenne et sécurisation des œuvres, accessibilité, gain de place et déplacement sécurisé en cas d'évacuation d'urgence. Mais, accordant une importance particulière à la notion de visibilité et d'esthétisme, elle a particulièrement insisté sur la nécessité de réaliser des conditionnements parfaitement « finis », de façon à restituer un aspect de qualité aux ensembles en réserves, dans un souci appuyé de valoriser le travail fait en coulisse lors de visites de personnes extérieures au musée. Cette exigence supplémentaire a été un peu difficile à intégrer au début par une équipe non habituée à travailler ni directement sur les collections ni dans cette perspective. Mais peu à peu, ce chantier a été l'occasion de repérer les talents de chacun et de les exploiter afin de permettre à chaque agent de trouver pleinement sa place...

\section{Quelques expériences phares}

Le chantier des collections a conduit l'équipe à mener des réalisations "phares" innovantes réduisant au maximum la part donnée au traditionnel et peu pratique... papier de soie froissé et aux mousses creusées, au rendu généralement soit peu fini soit très chronophage et prenant toujours beaucoup de place. Des solutions simples à réaliser ont été privilégiées, en compactant raisonnablement les collections tout en proposant un rendu très satisfaisant, tant en terme d'efficacité de calage, de gain de place, d'économie, de déplacement de collections et d'esthétisme...

\section{Cas des meubles sur mesures}

Le musée Crozatier intégrait dans son équipe des agents techniques particulièrement motivés par le projet. Leur savoir-faire les a donc conduits, suite aux réflexions menées avec Isabelle Boiché, à proposer des meubles métalliques à roulettes (fig. $\left.\mathbf{n}^{\circ} \mathbf{4}\right)$ permettant le déplacement aisé des collections d'une salle à l'autre. Une fois le modèle validé, ces «meubles-maisons» ont été réalisés pour recevoir des collections conservées sur des supports en mousse de polyéthylène superposables. L'équipe est même allée jusqu'à les équiper de fenêtres en polypropylène alvéolaires (voir photo ci-contre), pour mieux protéger les ensembles de l'empoussièrement tout en préservant la visibilité des collections! 
Figure 4

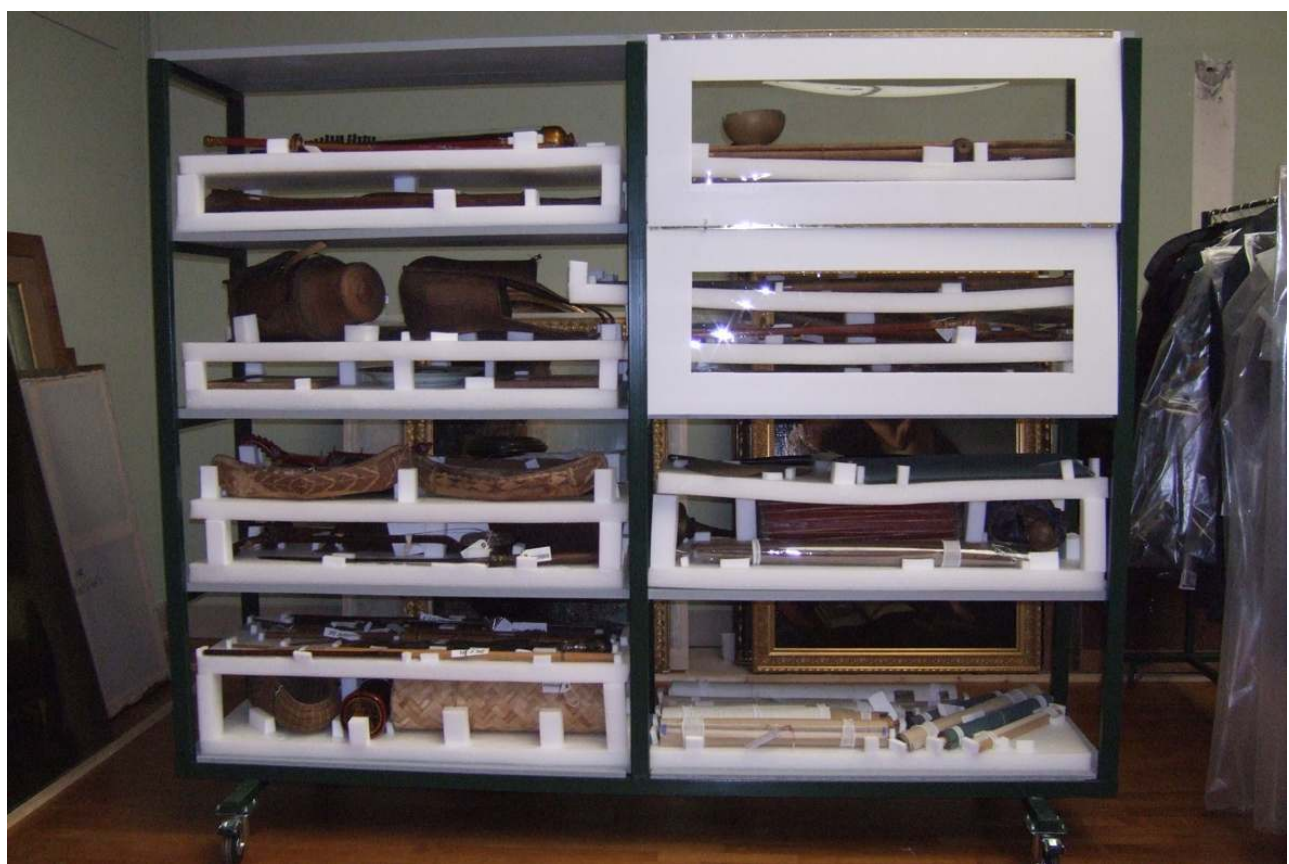

Meubles à roulettes « maison » et parois équipées de fenêtres.

(C) Boiché, Isabelle, IBM Conservation, 2010.

\section{Cas des boîtes à bannières}

41 Les mêmes agents ont aussi été formés par Isabelle Boiché à la fabrication de grandes boîtes à bannières (fig. $\mathbf{n}^{\circ} 5$ ). Ce travail a particulièrement intéressé l'équipe technique, le résultat aboutissant à la réalisation rapide de boîtes de grands formats ultra-légères ! 
Figure 5

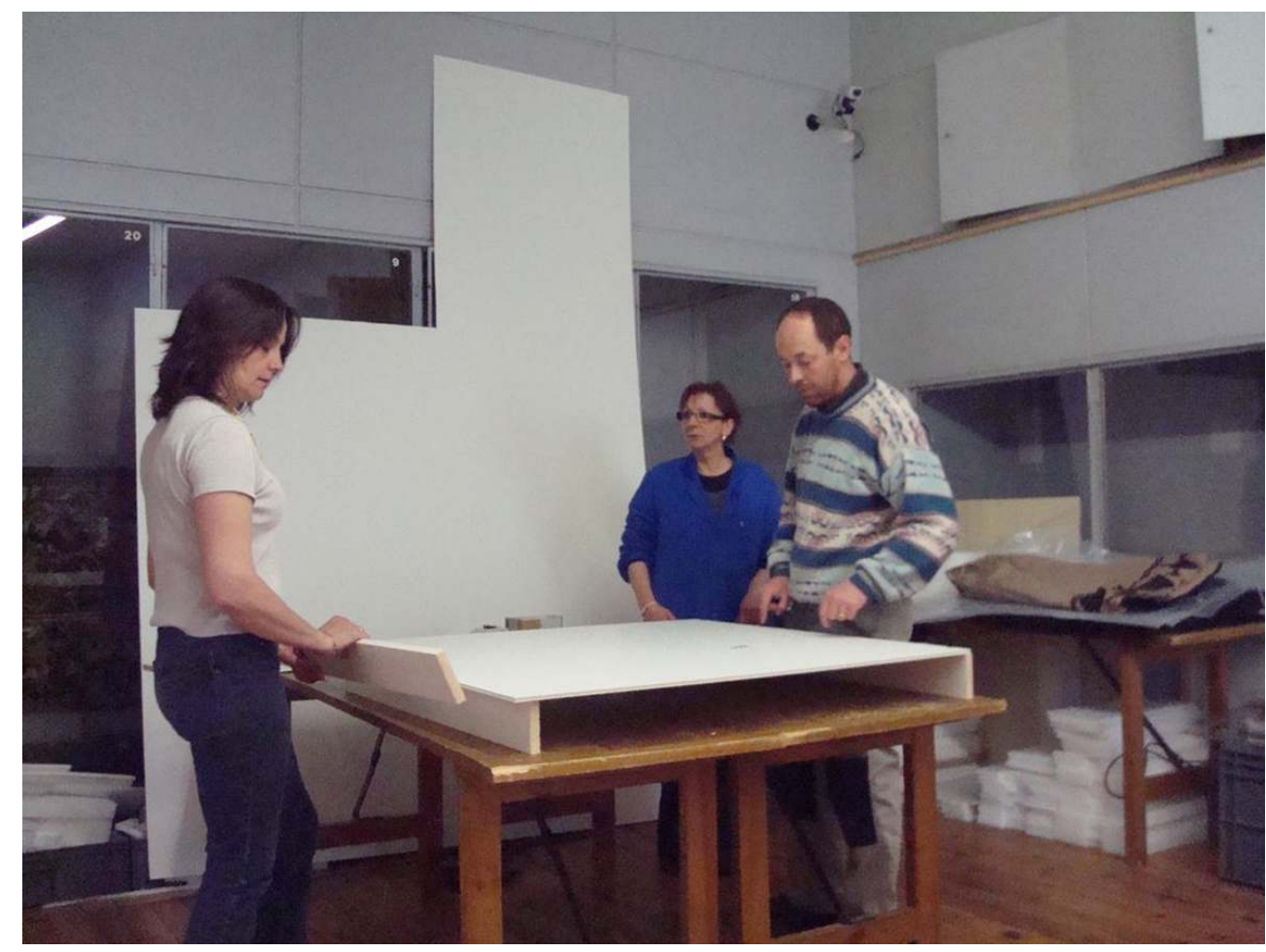

Fabrication de boîtes à bannières.

(c) Boiché, Isabelle, IBM Conservation, 2009.

\section{Cas des aménagements de tiroirs}

Dans les années 1990, un menuisier rattaché à l'équipe technique du musée avait réalisé de nombreux tiroirs de stockage de belle facture. Le chantier a conduit à la mise en teinte de ces tiroirs ${ }^{5}$ et à la fabrication de plateaux superposables en polypropylène alvéolaire et mousse $\mathrm{PE}^{6}$ et $\mathrm{PER}^{7}$ (fig. $\mathbf{n}^{\circ}$ ) afin d'exploiter au maximum ces volumes de rangement. Les céramiques et autres ensembles précieux y ont trouvé place, selon des modes de calage sécurisants, très esthétiques et très pratiques. 
Figure 6

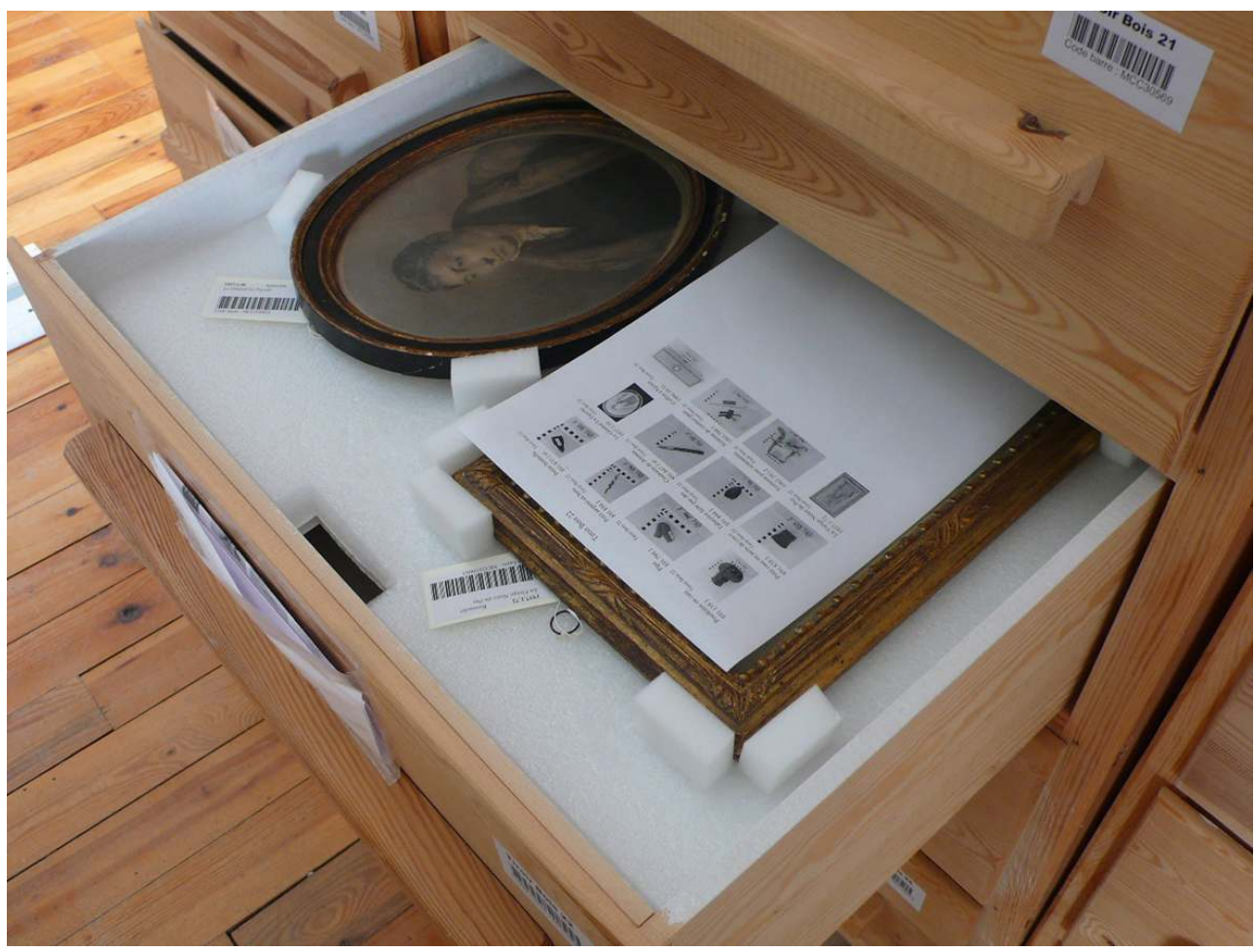

Aménagement interne de tiroirs.

(c) Boiché, Isabelle, IBM Conservation, 2010.

\section{Cas de la paléontologie}

Le chantier de conditionnement a commencé par le traitement de la collection de paléontologie. Le mode de contenant choisi a été la solution de bacs gerbables de type "Norme Europe" à fond et paroi pleins ${ }^{8}$ installés dans des structures porteuses métalliques légères ayant l'avantage de transformer ces bacs en «tiroirs" pour le rangement définitif.

Ces collections, fragiles, ont été calées au sein des bacs (fig. $\mathbf{n}^{\circ} \mathbf{7}$ ), sur des supports sur mesure en mousse de polyéthylène réticulée (PER) non abrasive dont les modalités de mise en forme ont ensuite été systématisées à l'ensemble des collections 3D. 
Figure 7

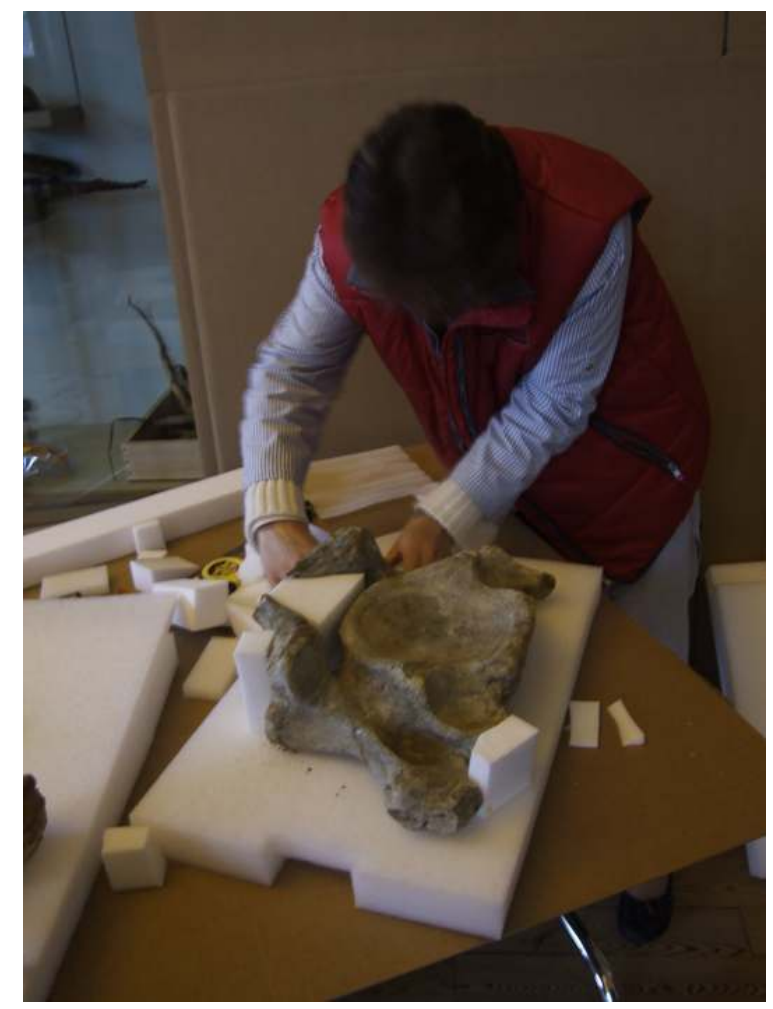

Réalisation de plateau de calage avant mise en bacs.

(c) Boiché, Isabelle, IBM Conservation, 2009.

Pour les ensembles moins fragiles, de simples alvéoles en mousse de polyethylène (PE), soudées ou amovibles selon les cas, ont pu également être adoptées.

\section{Cas des Naturalia}

Le conditionnement des Naturalia (animaux naturalisés : mammifères, oiseaux, poissons, etc.) a été l'occasion de réfléchir sur des solutions variables en fonction des formats des pièces.

Dans ce cadre, les petits oiseaux ont été calés dans leur carton à l'aide de "rails » en mousse à l'intérieur desquels étaient glissés les socles en bois : cette solution permettait de bien caler chaque pièce et d'éviter le traditionnel calage en papier de soie froissé, rendant invisible les oiseaux et ne permettant aucun contrôle. Ces solutions pourront d'ailleurs être conservées comme plateau de conservation et de déplacement, une fois les ensembles redéployés sur des étagères dans les réserves définitives.

Tous les grands formats ont été calés sur des supports individuels en mousse PE puis mis en cartons (fig. $\mathbf{n}^{\circ} \mathbf{8}$ ) ; certains cartons ont d'ailleurs été équipés de "portes " afin de permettre une ouverture frontale facilitant le contrôle et l'extraction de pièces particulièrement fragiles. 


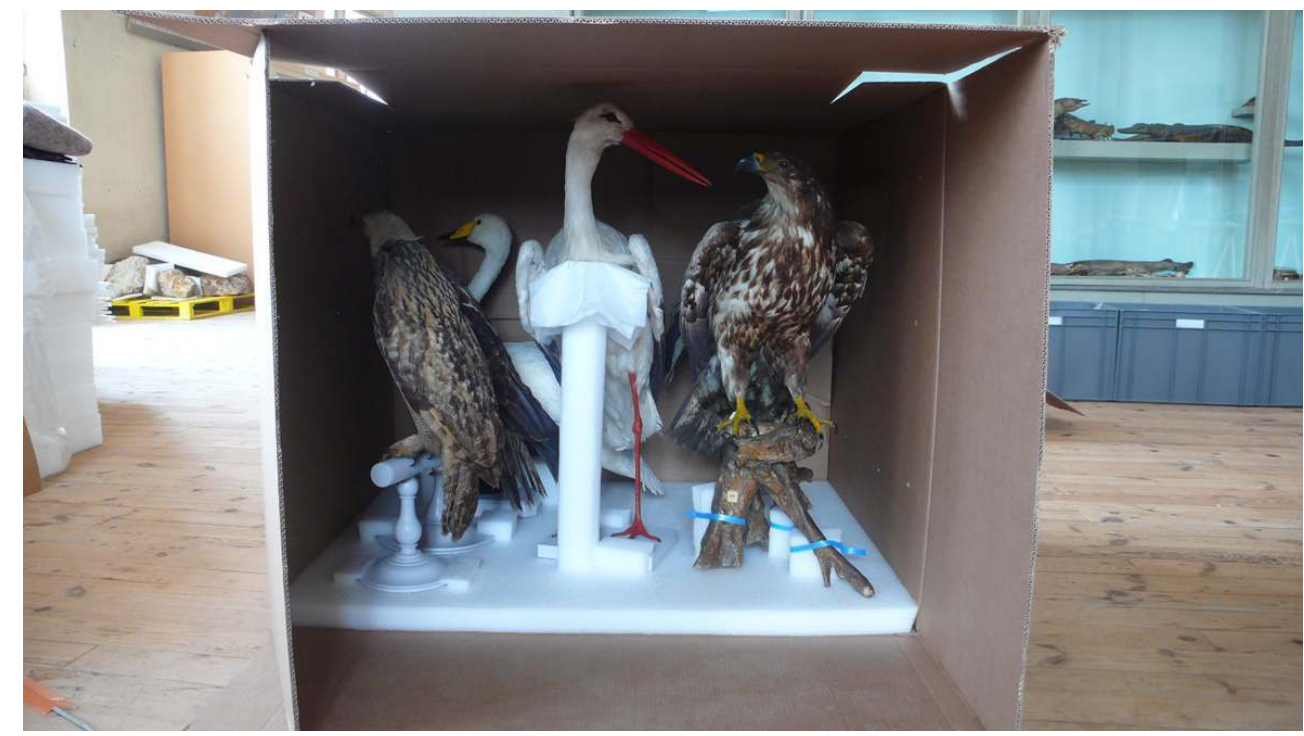

Oiseaux mis en carton.

(c) Boiché, Isabelle, IBM Conservation, 2009.

\section{Cas des échantillons de dentelles}

49 Quelques milliers d'échantillons de dentelles ont été dépoussiérés puis mis en pochettes transparentes directement soudées en interne, à partir de formats standard prédéterminés.

\section{Cas du lapidaire}

Le lapidaire a été placé sur des palettes norme européenne ignifugées et traitées contre les infestations. Cela représente environ 60 palettes qui, pour des raisons de commodité dans la gestion future du chantier, ont été transférées dans un local loué pour l'occasion.

\section{Cas des tableaux}

51 L'emballage et le déplacement des collections du musée devaient en grande partie être assumés en interne à l'aide de matériel et d'engins de circulation disponibles au sein des services municipaux. Quelques pièces, difficilement accessibles et de grands formats, ont toutefois été confiées à un transporteur spécialisé dans le domaine des œuvres d'art. Ainsi, les tableaux de très grand format exposés dans l'escalier monumental ont été décrochés par un prestataire ${ }^{9}$ expérimenté sous la supervision de deux restaurateurs. Un échafaudage a été installé par ce transporteur qui, en trois jours, à réussi, à l'aide des agents techniques du musée, à décrocher cinq tableaux qui ensuite ont été examinés puis roulés (fig. $\mathbf{n}^{\circ} \mathbf{9}$ ) par les restaurateurs ${ }^{10}$. 
Figure 9

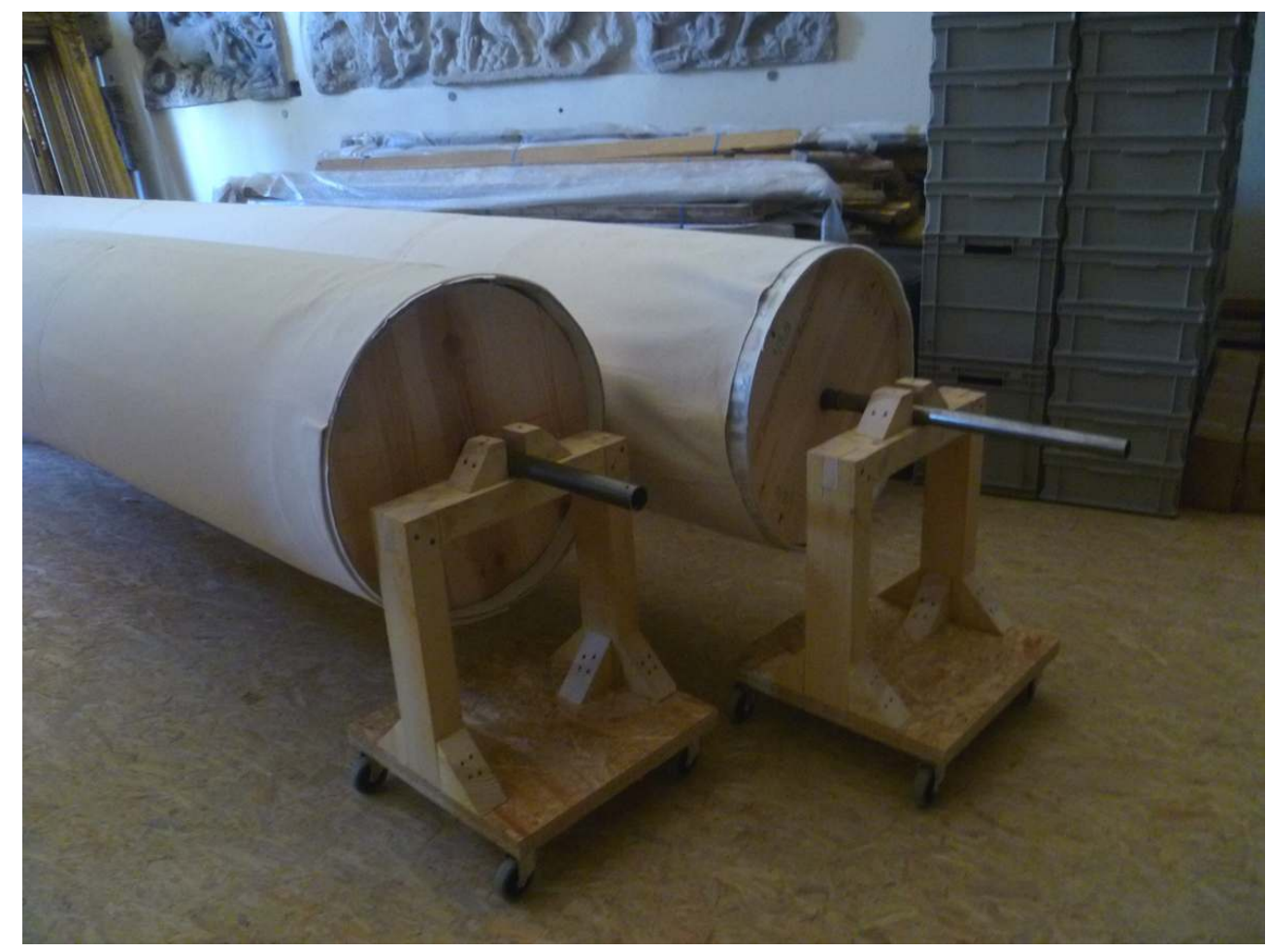

Tableaux grands formats roulés.

(c) Boiché, Isabelle, IBM Conservation, 2011.

52 Les tableaux sans cadre ont bénéficié, après examen et dépoussiérage, de la systématisation de dos protecteurs en plaques de polypropylène de façon à pouvoir ensuite les ranger verticalement en toute sécurité dans des meubles. Certains ont pu préalablement être consolidés provisoirement par un restaurateur (pose de facing).

\section{Quelques chiffres}

53 Ce chantier a permis de redécouvrir une collection de près de 60000 items ! Parmi eux :

- 880 tableaux ont été équipés de dos protecteurs

- 3000 dentelles ont été conditionnées en pochettes Mélinex ${ }^{\circledast}$

- 933 spécimens de zoologie ont été traités

- 848 céramiques ont été calées

- 122 verreries ont été traitées

- 6051 documents d'arts graphiques reconditionnés dans des pochettes de Mélinex ${ }^{\circledast}$

- 1262 objets et documents des civilisations extra-européennes

- 1629 objets d'artisanat local

- etc. 


\section{Achèvement du chantier des collections, premiers pas vers le chantier de rénovation}

Une fois le chantier des collections achevé, il a fallu penser à l'imminence des travaux... et au maintien des collections dans un site à rénover. La perspective d'évacuer les collections n'étant plus d'actualité depuis bien longtemps, il avait été convenu avec l'équipe d'architectes chargée du projet d'installer les collections au rez-de-chaussée puis d'orchestrer la migration progressive des collections au fil du phasage des travaux. Tous les conditionnements avaient été réalisés en intégrant cette contrainte majeure.

Dans cette perspective, tous les ensembles ont été mis en boîtes, bacs ou caisses et sous film polyane. Des équipements « maison » ont été imaginés pour compresser au mieux les collections dans des espaces réduits (fig. $\mathbf{n}^{\circ} \mathbf{1 0}$ ) en les sécurisant le mieux possible.

Figure 10

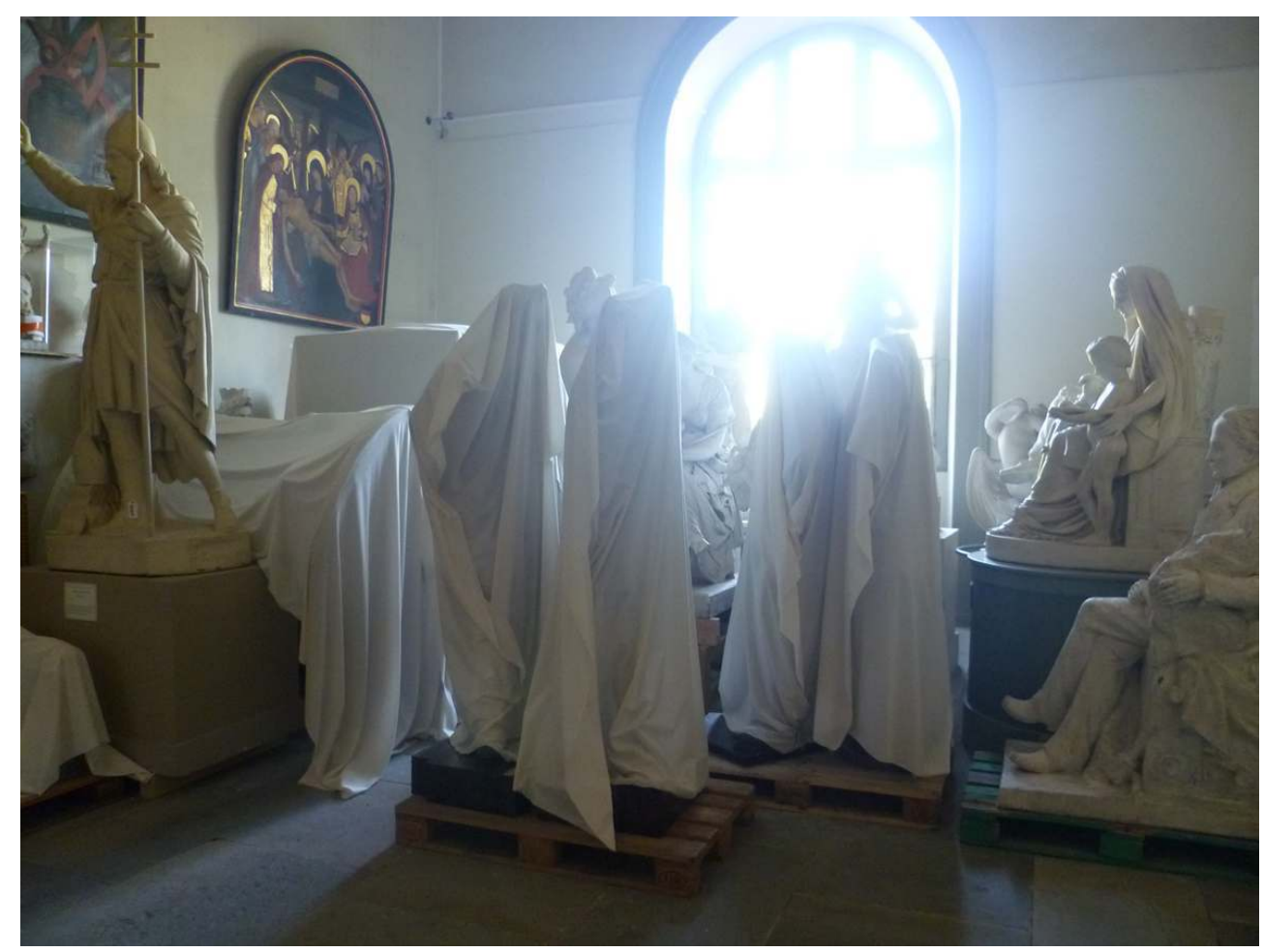

Des collections regroupées en vue du chantier de rénovation.

(c) Boiché, Isabelle, IBM Conservation, 2011. 
Figure 11

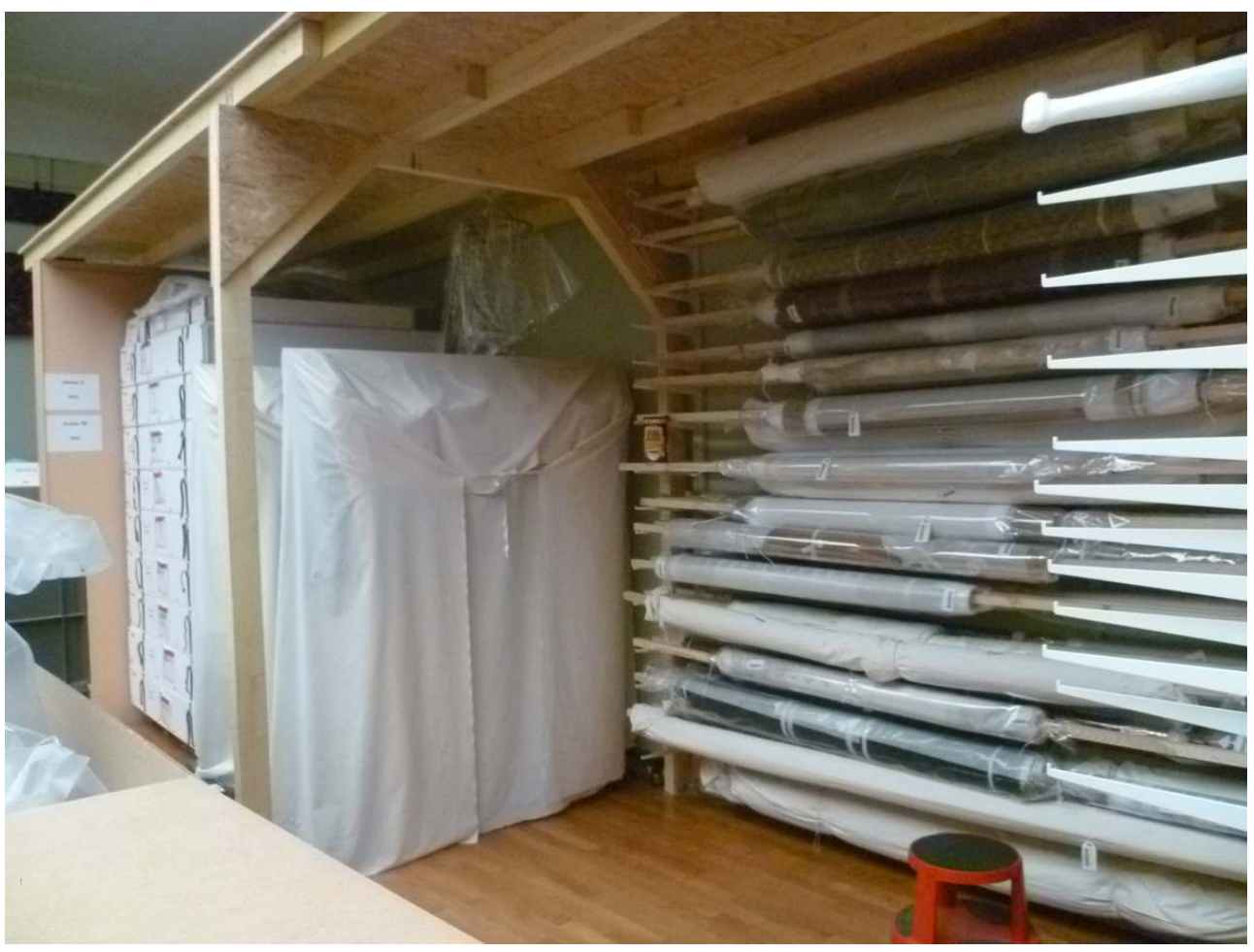

Des collections regroupées en vue du chantier de rénovation.

(c) Boiché, Isabelle, IBM Conservation, 2011. 


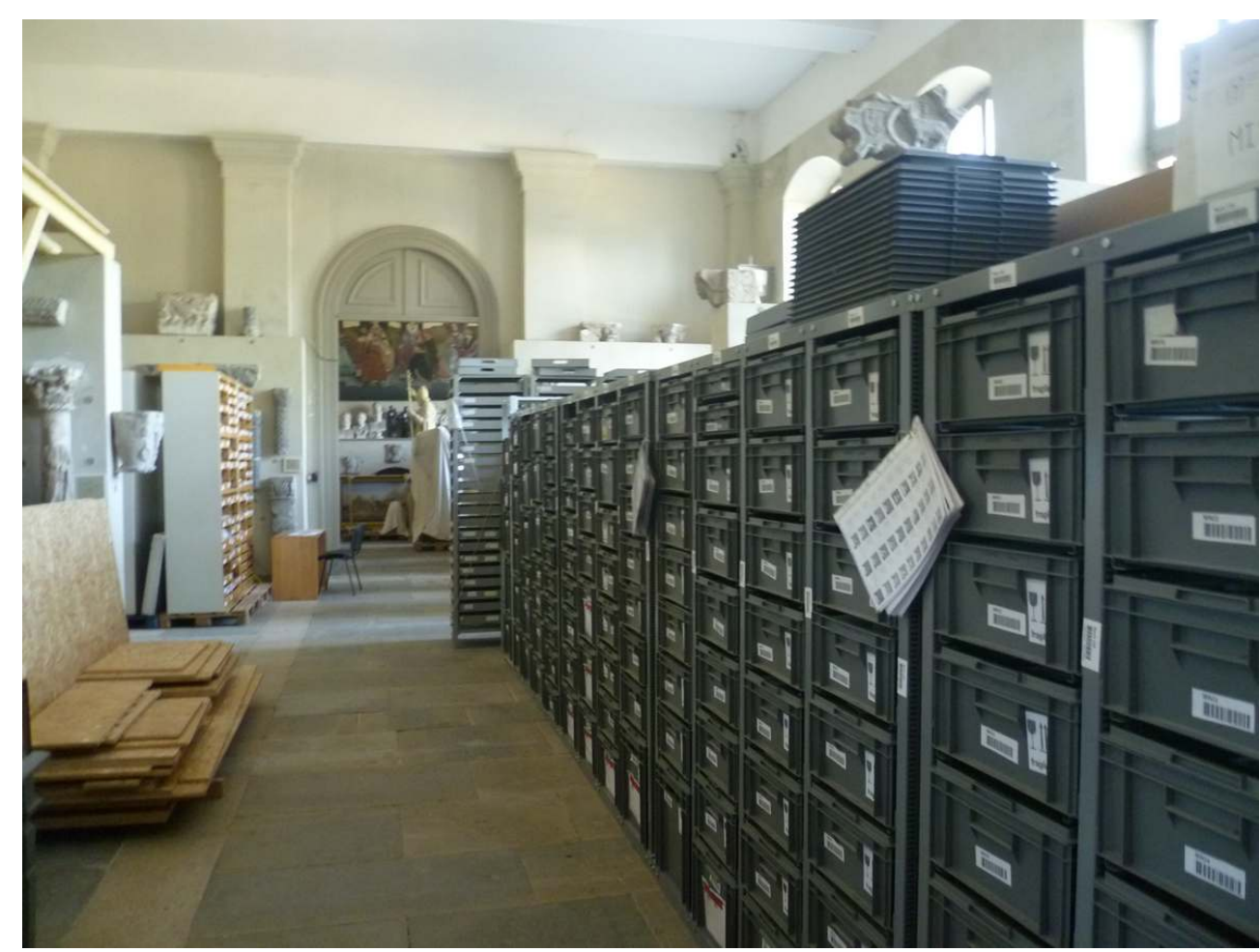

Des collections regroupées en vue du chantier de rénovation.

(c) Boiché, Isabelle, IBM Conservation, 2011.

\section{Un chantier en interne : avantages et inconvénients}

Le musée Crozatier a opté en 2007 pour la réalisation d'un chantier en interne, sans doute parce qu'à l'époque l'externalisation d'un chantier n'était guère envisageable, faute d'expérience sur le sujet, mais surtout parce que le responsable de l'institution souhaitait une réelle implication de son équipe dans le projet global de rénovation, quitte à le programmer très en amont des travaux et sur plusieurs années.

57 La mise en place d'un chantier de cette ampleur requiert néanmoins quelques impératifs élémentaires :

58 - L'équipe doit être en nombre suffisant pour répondre à la masse de travail s'étalant sur de nombreuses années.

59 - Les agents doivent être motivés et... doivent le rester: la charge de travail est importante, correspondant d'ailleurs rarement aux fiches de postes de travail initiales.

60 - L'équipe doit être stable.

61 - Le prestataire doit savoir maîtriser la notion de "management» pour inculquer de nouvelles formes de travail et de suivi de ce travail.

62 - Le prestataire doit avoir une approche « économique » du projet et savoir proposer des solutions adaptées mais plaisantes à mettre en œuvre: le découragement peut en effet contaminer le moral de l'équipe, il importe donc de toujours trouver des objectifs et arguments pour valoriser le travail fait et maintenir le cap dans la meilleure ambiance 
possible. Il importe donc de fractionner au maximum les objectifs pour diversifier les missions, et avancer progressivement sans toutefois perdre de vue les objectifs finaux...

- Le prestataire doit savoir intégrer dans les nouvelles habitudes de travail l'acceptation de la notion de " travail à la chaîne ", « stocks » et «standardisation » pour travailler en toute efficacité, sans dispersion ni perte de temps ou gaspillage de fournitures et d'énergie.

64 - L'équipe doit participer à l'établissement des procédures afin de mieux adhérer à cellesci.

65 - Le prestataire doit réaliser un suivi qualitatif sans oublier de maintenir une vraie motivation des troupes, le «relationnel» étant sans aucun doute une des clefs de la conservation préventive...

66 - Le suivi quantitatif ne doit pas être laissé de côté même s'il est "spontanément» souvent mal vécu au début; il importe que le prestataire et le responsable de l'institution sachent amener l'équipe à accepter l'idée qu'il est important de quantifier régulièrement le nombre d'objets traités, non dans un esprit de "stricte surveillance » mais afin de rendre des comptes précis sur le travail conduit en coulisses, dans un souci de pouvoir, ensuite, mettre en avant la masse de travail développée durant ces nombreux mois...

\section{Perspectives}

67 En tout, près de 15 personnes ont participé à différentes phases de ce chantier, ce qui correspond à 272 mois soit 5440 jours ou environ 45000 heures de travail pour traiter quelques 59613 items.

Une page se tourne définitivement, au bénéfice de grands travaux dont nous entendrons sans nul doute parler, l'inauguration du musée rénové étant programmée pour la fin de l'année 2014.

Gageons que ce travail saura produire tous ses effets au sein de réserves rénovées dans un outil moderne de protection, de conservation et de diffusion culturelle dans un souci de développement durable affirmé, concept émergeant dans la littérature patrimoniale mais véritable « $\mathrm{ADN}$ » d'un concept vieux maintenant de plus de 20 ans en France : celui de la conservation préventive...

\section{Les mots de la fin... ou paroles d'acteurs du chantier des collections}

La période du chantier des collections s'achève, c'est l'heure du bilan avec les agents qui en ont été les acteurs.

La satisfaction n'est pas feinte, et bien méritée, mêlée de fierté à la vue des rayonnages : «Ça n'a pas du tout la même allure qu'avant ! »

Cette satisfaction professionnelle relève des trois ordres : savoirs, savoir-faire, et savoir être. 
Sur le plan intellectuel, avec le bagage de formations théoriques et pratiques sur les matériaux et la conservation préventive, les agents ont acquis les connaissances nécessaires au choix et à la conception des divers conditionnements. Ils en ont maîtrisé l'élaboration et la fabrication, ainsi que celles de la chaîne du traitement des objets : dépoussiérage, photographie, informatisation des fiches, et enfin conditionnement.

Le travail sur certaines collections est jugé plus valorisant : le traitement des objets 3D a davantage fait appel à leur ingéniosité, tandis que le dépoussiérage de milliers d'échantillons de dentelles est apparu un peu lassant. Quant aux « fotus et aux squelettes, c'était pas très chouette »...

Cette fréquentation « intime » des collections, et la connaissance qu'ils en ont tirée leur a apporté un sentiment d'appartenance plus fort à une équipe et à une institution : «On avait le sentiment d'être plus dans le musée ».

L'esprit d'équipe a été grandement renforcé par l'adhésion de tous aux nouvelles missions du chantier des collections. Toute l'équipe de surveillance et d'entretien s'est vue confier des tâches dans la mesure des compétences de chacun, et l'équipe de l'atelier s'est également investie, dans la manutention des objets lourds ou volumineux, et dans la conception et la fabrication de meubles de rangement.

L'idée d'avancer vers un but, et de mesurer les résultats, par exemple au vu de la diminution des espaces occupés par les collections, renforce la satisfaction et l'esprit d'équipe.

Les mots qui reviennent dans leurs propos, et qui rendent hommage à leur action sont « actif, participation, impliqué et initiative ».

Martine : « C'était une expérience très enrichissante, maintenant je préfère être en chantier qu'en surveillance. »

Gilles : « On a appris des choses, c'était motivant. »

Patricia (récolement) : «Je suis impressionnée de voir tous les objets disparus qu'on a relocalisés. »

Olivier (Atelier) : « C'est un gros travail d'équipe, de longue haleine, qui nous a fait cogiter, trouver des solutions. On a participé à quelque chose de bien, on ne rénove pas un musée plusieurs fois dans sa carrière. »

Édith (suivi photos et site musée) : «J'ai été impressionnée par deux choses : les capacités des collègues à mener ce chantier à bien, et l'immense variété des collections. »

Monique : « Ça m'a appris beaucoup de choses, comment on conserve les objets dans les réserves.»

Radhia : «J'ai tout fait, tout essayé : les dentelles, les pièces, la mousse, les caisses... » Isabelle (mandataire) : « un chantier humainement exceptionnel ; des énergies fortes durant tous ces mois passés à œuvrer en coulisses... Je crois que j'ai réussi à les 
contaminer... à leur transmettre le virus de la conservation préventive... Et à leur donner envie... de se faire plaisir!»

\section{NOTES}

1. - Pour l'histoire du musée nous renvoyons au catalogue : GRANDJEAN, Gilles (dir). Histoire du musée Crozatier : Le Carrosse et la momie, mais encore... Le Puy-en-Velay : Musée Crozatier, 2002.

2. - Restauratrice de tableaux.

3. - Restauratrice de collections ethnographiques et consultant en conservation préventive.

4. - Plus de 52000 objets ou lots d'objets.

5. - Intérieur peint à l'acrylique.

6. - Polyéthylène.

7. - Polyéthylène réticulé.

8. - Plus coûteux que des bacs à parois ajourées mais plus protecteurs contre l'empoussièrement.

9. - Société BOVIS.

10. - Christian Morin et Vélia Dahan.

\section{RÉSUMÉS}

Chantier de collection... Dépoussiérage de collection... Conditionnement de collection... Marquage de collection... Prise de vue... Informatisation... des mots clefs ancrés depuis maintenant quatre ans dans le quotidien d'un musée qui entre enfin en 2012 dans sa phase active de rénovation architecturale! Le musée Crozatier a fermé ses portes aux visiteurs en juin 2011, sa restructuration étant programmée de 2012 à 2014. Dans cette perspective, les troupes du musée ont été rassemblées depuis de nombreux mois pour conduire, avant même sa rénovation, un chantier de grande ampleur dans une logique implacable de conservation préventive... En effet, le musée Crozatier prépare depuis plusieurs années une reconversion en profondeur pour présenter au public une vision modernisée de son parcours muséographique en intégrant, préalablement à ce projet, une démarche de remise à niveau fondamentale pour mettre ses collections dans des conditions de conservation performantes. À l'origine de cette impulsion, un conservateur, Gilles Grandjean, soucieux d'améliorer concrètement des «coulisses patrimoniales » pas assez performantes face au courant de professionnalisation qui colonise peu à peu les coulisses des musées depuis les années 1990. En préalable à cette rénovation, le musée et la communauté d'agglomération du Puy-en-Velay ont sélectionné, par le biais d'un marché public, un prestataire expérimenté et spécialisé dans le domaine de la conservation préventive. Dans ce cadre, l'équipe du musée portée par le projet s'est immédiatement montrée soucieuse de changer ses habitudes de travail en intégrant des procédures de travail innovantes, modifiant en profondeur les missions... 
Collections renovation... cleaning museum objects, re-packing of collections... Marking of collections... photography... digitization of collections... are some of the key words that have been anchored in the daily activities of a museum, which, at last, enters, in 2012, in an active phase of architectural renovation! The Crozatier Museum closed its doors to the Public in June 2011, its renovation will take place between 2012 and 2014. With this project in mind, and already before its proper renovation, the Museum formed a team several months ago to undertake an extensive cataloguing and re-packing project that draws on a rigorous preventive conservation strategy. Indeed, the Crozatier Museum has been involved over the past years in a profound restructuration in order to offer the Public a modernized vision of its museographic trail and before doing so, it opted for a profound change to ensure the best conservation conditions for its collections. The driving force behind this change comes from a curator, Gilles Grandjean, who aimed to manage more efficiently heritage collections in the context of the increasing professionalization which colonized museums, behind the scene, during the 1990s. An experimented professional in the sector of preventive conservation was selected, through public procurement contract, by the Museum and an agglomeration community (a metropolitan government structure) to provide assistance for the renovation. In this context, the Museum team embraced the project and committed itself to change working habits in order to adopt innovating working processes and radically transform its missions... (Carine Durand)

\section{INDEX}

Mots-clés : conservation préventive, chantier de collection, récolement, formation de personnel, visibilité, développement durable

\section{AUTEURS}

\section{ISABELLE BOICHÉ}

IBM Conservation (Bourgoin-Jallieu, Isère), consultant en conservation préventive ayant encadré le chantier des collections du musée Crozatier entre 2008 et 2011 Isabelle.boiche@free.fr

\section{EMMANUEL MAGNE}

attaché de conservation au musée Crozatier emmanuel.magne@mairie-le-puy-en-velay.fr 